\title{
Multiple robot operations for maritime search and rescue in euRathlon 2015 competition
}

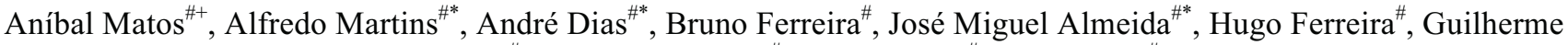 \\ Amaral $^{\#}$, André Figueiredo ${ }^{\#}$, Rui Almeida ${ }^{\#}$, Filipe Silva ${ }^{\#}$ \\ "INESC TEC - INESC Technology and Science \\ Rua Dr. Roberto Frias, Porto, Portugal \\ *ISEP - School of Engineering, Polytechnic Institute of Porto \\ Rua Dr. Antonio Bernardino de Almeida, 431, Porto, Portugal \\ ${ }^{+}$FEUP - Faculty of Engineering Porto University \\ Rua Dr. Roberto Frias, Porto, Portugal
}

\begin{abstract}
This paper presents results of the INESC TEC participation in the maritime environment (both at surface and underwater) integrated in the ICARUS team in the euRathlon 2015 robotics search and rescue competition. These relate to the marine robots from INESC TEC, surface (ROAZ USV) and underwater (MARES AUV) autonomous vehicles participation in multiple tasks such as situation assessment, underwater mapping, leak detection or victim localization. This participation was integrated in the ICARUS Team resulting of the EU funded project aimed to develop robotic tools for large scale disasters. The coordinated search and rescue missions were performed with an initial surface survey providing data for AUV mission planning and execution. A situation assessment bathymetry map, sidescan sonar imaging and location of structures, underwater leaks and victims were achieved, with the global ICARUS team (involving sea, air and land coordinated robots) participating in the final grand Challenge and achieving the second place.
\end{abstract}

\section{INTRODUCTION}

Search and rescue (SAR) is a relevant area of application and research for the use for autonomous and robotic systems. The problems arising from the necessity of fast response, possible human risk in the rescue activities and sheer dimension (both in areas to cover, information to be gathered and intervention to be performed) of the problems suggest and stress the benefits on the use of robots to assist in search and rescue operations. Robots can play an important role either by allowing the human intervention in disaster areas with reduced risk, providing valuable information and assessment with efficient resources, computer aided detection or simply extending the coverage.

This paper presents the results of the INESC TEC participation in the maritime environment (both at surface and underwater) integrated in the ICARUS team in the euRathlon robotics search and rescue competition.

The ICARUS (Integrated Components for Search and Rescue) project [1] is an EU funded research aiming to develop robotic tools for search and rescue operations. It addresses large scale disaster scenarios, both in urban and in maritime environments. The integration of aerial, maritime and land based robotic tools is also under consideration in the ICARUS context, both from an operative point of view and from technical interoperability aspects.

As a partial results of the project a set of robotic competencies for SAR have been developed, and in view of this, a team was formed to participate in the euRathlon 2015 [2] robotic competition.

This competition is an international robot completion focusing on multi-domain (air, land and sea) search and rescue. It was also funded by EU to drive and benchmark robotics research in real world relevant rescue scenarios, being the first technically challenging robotics competition specifically addressing cooperation of heterogeneous air, land and sea robots.

The ICARUS team participated with a complete team covering all the domains with aerial, land and maritime robots integrated in a common infrastructure and addressing the challenge in cooperative way.

The search and rescue area of application in robotics has been subject of active research and developments [3]. These range from particular topics such as robot deployment in outdoor scenarios [4] or target detection techniques [5] to specially designed robots [6] or large scale projects [1], [7].

In the marine environment two types of problems arise: either single (or low number) of human victims search from incidents such as man-over-board or ship wrecking, or, large scale disasters as the Costa Concordia[8] or natural events as hurricanes [9] or the Fukushima incident [10]. 
Robotic competitions can provide near to real world testing environments and benchmarking. In the search and rescue, Robocup Rescue [11] is a good example in urban oriented scenarios and euRathlon [2] in outdoor environments. euRathlon in particular takes inspiration on the Fukushima incident and presents a real world scenario involving both marine, aerial and land robotics (both outdoor and indoor). The 2015 edition that occurred in September 2015 in Piombino, Italy, was the first time that land, air and marine robots performed a life-like mission with a common goal. The competition has a set of particular challenges covering mapping, victim detection, situation awareness and intervention (manipulation of valves) in the three environments (air, land and water), culminating in a Grand Challenge where robots must operate in coordination in all environments.

\section{EURATHLON ROBOT COMPETITION}

The euRathlon competition intended to present a real world search and rescue scenario for the use and benchmarking of multiple domain (air, land a sea) cooperating robotic tools.

It was inspired in real disaster events as the Daichi power plant disaster in Fukushima, where a large scale disaster occurred (tsunami and nuclear reactor leak) with hard limitations on human intervention. This example clearly evidenced the need for robotic tools in search and rescue for diverse tasks such as surveying, situation assessment, inspection, mapping of damages, victim search and even remote intervention.

In order to present an incremental challenge and allowing multiple technological capabilities testing, the competition was developed with multiple trials leading to increased complexity and requiring increased multiple system cooperation. These partial challenges and trials consisted in:

- Sub-Challenge $(\mathbf{L}+\mathbf{A})$ : Survey the building and search for a missing worker.

- Sub-Challenge $(\mathbf{S}+\mathbf{A})$ : Pipe inspection and search for a worker.

- Sub-Challenge $(\mathbf{L}+\mathbf{S})$ : Stem the leak

- Land Trial (L1): Reconnaissance in urban structure

- $\quad$ Land Trial (L2): Mobile Manipulation (stopcock open/closing)

- Sea Trial (S1): Navigation and environmental survey

- Sea Trial (S2): Leak localisation and stopcock open/closing.

- $\quad$ Air Trial (A1): Aerial detection and mapping

- Air Trial (A2): Aerial reconnaissance inside a building

The individual trials addressed specific tasks in a single domain and the sub-challenges introduced multi-domain robot cooperation. All these culminated in the Grand Challenge where a complete team with marine, air and land robots would intervene in the disaster scenario providing not only a complete initial assessment and mapping of all environment (both outdoor and indoor land and also marine underwater), identification of relevant targets and damage assessment, victim location (in land and at sea) and finally coordinated manipulation indoor and undersea.

The competition took part in Piombino, Italy from 17 to 25 September 2015, in a near sea location next to a power plant.

The scenario involved the Torre del Sale building, a ruin next to the beach, the nearby beach and land surroundings and a small port with the underwater environment area (Figure 1).

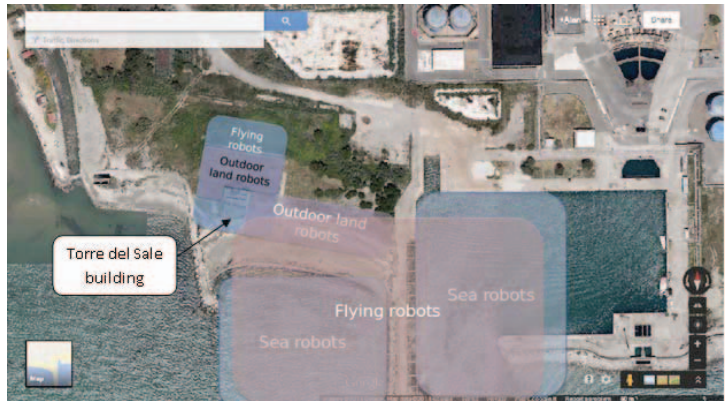

Fig. 1 - Competition scenario

This scenario setup closely mimics the Fukushima event, with a disaster in and industrial or nuclear reactor (represented by the Torre del Sale building) close to the sea and with nearby sea infrastructures (a port structure) that need to be surveyed. Aerial robots should be able to provide coverage, survey and mapping of all area, and indoor assessment, land robots would provide close range inspection and actuation (such as valve manipulation) and marine robots allow for actuation, search and survey in the underwater environment.

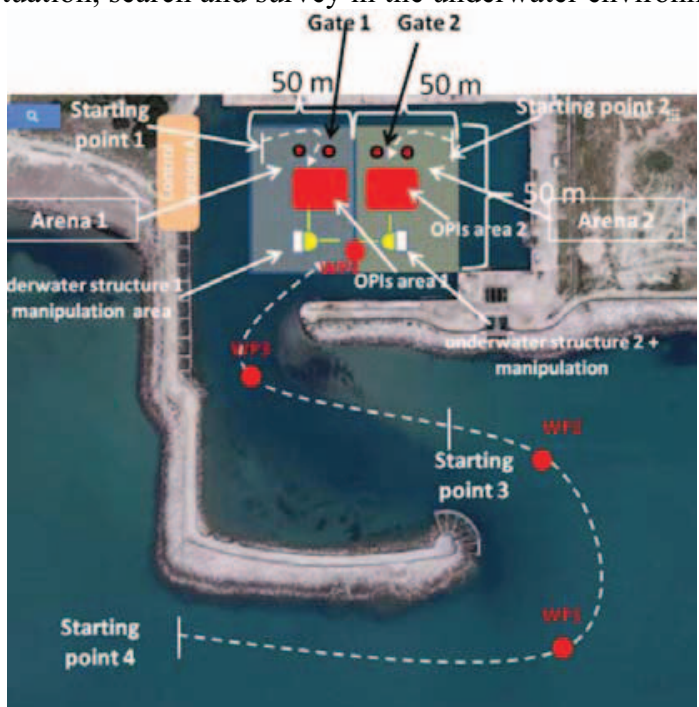

Fig 2 - Maritime competition scenario (from [12])

The marine scenario consisted of two competition areas (allowing multiple team simultaneous runs) with submerged structures, pipes and eventually victims in the nearby port. It 
also covers the sea access area (Figure 2) for autonomous navigation.

The work described in this paper addresses the marine scenario search and rescue part $(\mathrm{S} 1, \mathrm{~S} 2, \mathrm{~L}+\mathrm{S}, \mathrm{A}+\mathrm{S}$ and Grand Challenge) of the ICARUS team that tackled all the challenges and the final Grand Challenge. The marine robots used were an unmanned surface vehicle and an autonomous underwater vehicle from INESC TEC.

The sea trials cover several tasks useful for search and rescue underwater operations. These are: demonstration autonomous navigation capabilities and area survey and mapping, underwater infrastructure damage assessment and leak detection, underwater victim detection and localization and underwater manipulation, namely closing of a valve.

The cooperative challenges, cover both the cooperative search for victims, global situation assessment and leak detection and repairing.

In the underwater scenario there are multiple submerged infrastructures and pipes that depending on the specific trial need to be identified and characterized. Piping leak are represented by specific markers, and for victim identification, test dummies are placed in unknown locations. Although the general operation scenario is unknown, prior to the challenges details on the state positioning and possible location of targets are changed.

\section{MARITIME MULTI-ROBOT ROBOT TEAM}

\section{A. ROAZ Autonomous Surface Vehicle}

One of the robotic platforms used in the euRathlon completion in the marine scenario was the autonomous surface robot ROAZ (Figure 3). This vehicle performed the missions in cooperation with the MARES autonomous underwater vehicle (Figure 4).

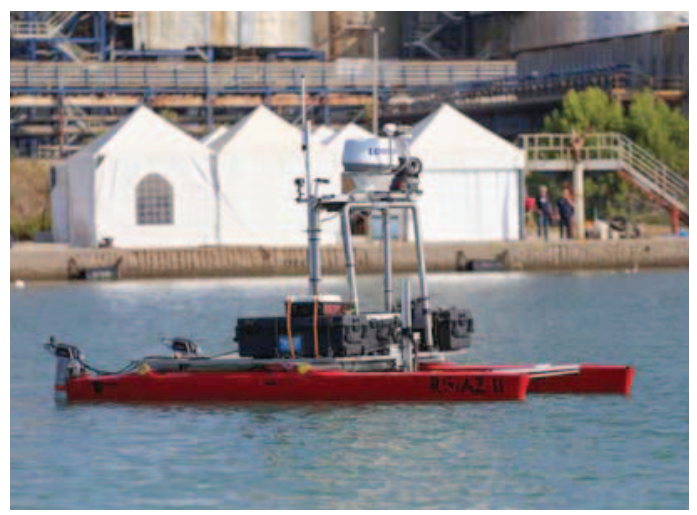

Fig. 3 - ROAZ USV

ROAZ is a robotic unmanned surface vehicle developed as a testbed for research and development on marine robotics. It has performed a large number of operational missions in the sea. It was also one of the maritime robotic platforms used in the ICARUS sea demonstration to deploy robotic liferafts [13].
It is an electric powered $4.2 \mathrm{~m}$ long catamaran capable of autonomous operation up to $8 \mathrm{hrs}$ and with onboard computer navigation and control. It is equipped with a precision dual band RTK GPS receiver and inertial navigation system providing not only precise location for navigation purposes but also accurate geo-referencing of sensory data.

Above water is equipped with a thermographic and a visible spectrum cameras (with on board processing that can be applied in search operations [14]) and a RADAR used for large obstacle detection. In addition for close range environment 3D modeling and obstacle detection the system can be equipped with a 3D Velodyne LIDAR.

The robotic vehicle is also equipped with a single beam echosounder, an Imagenex DeltaT multibeam sonar and an Imagenex Sportscan side scan sonar. These sonars are used for underwater environment modeling and bathymetry tasks. In the euRathlon competitions they were used sea bottom modeling and imaging.

For above water communications when the vehicle is within range, ROAZ is equipped with an IEEE 802.11a link.

The on board computer is responsible for the autonomous mission control, and can be used to process the vehicle sensors in order to detect relevant events or perform sensor oriented missions.

\section{B. MARES}

MARES is an autonomous underwater vehicle developed for marine environment remote monitoring, survey and modeling.

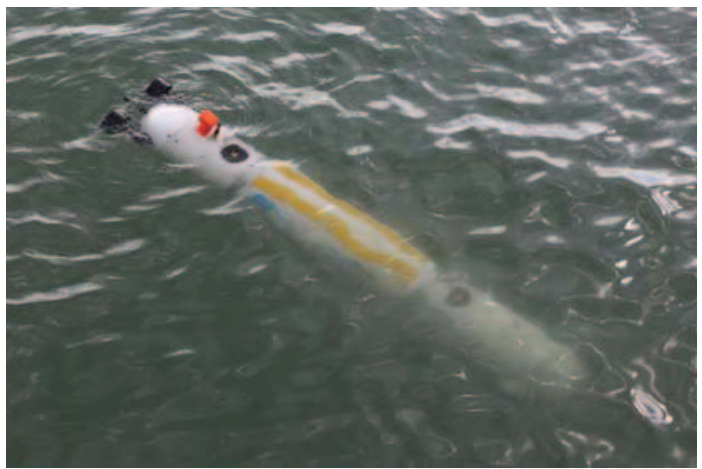

Fig. 4 - MARES AUV

It is a $2 \mathrm{~m}$ long, $20 \mathrm{~cm}$ diameter circular shape (Figure 4 ) vehicle. It has a modular design with multiple possible sections with application sensors and systems. This AUV has a high maneuverability with two stern mounted forward thrusters and two vertical ones, allowing for complete pitch control. This has the advantage in comparison with traditional fin based systems of high control at low speeds, excellent bottom following characteristics and good control for visual inspection tasks.

MARES is equipped with on board INS, and compass and can navigate in a LBL acoustic network. It has onboard processing and control in a $\mathrm{PC} 104 \mathrm{CPU}$ running modular 
software in a Linux based system. For the euRathlon mission it was equipped with a color camera mounted on the aft looking at a slanted angle downwards.

When at surface it can receive GPS position information in an antenna fin that also carries a Wifi antenna for mission downloading and information retrieval.

\section{SEARCH AND RESCUE MISSION}

\section{A. Environment initial survey and mapping}

The SAR mission is performed by the ASV and AUV taking advantage of each vehicle capabilities.

Both vehicles have the challenge of identifying the underwater points of interest inside the predefined areas in a cooperative perception manner. Moreover, the heterogeneity of the vehicles and the sensors payload of each one, provides complementary information to the SAR mission objectives.

The global mission approach is composed by three steps: an environment initial survey, performed by the ROAZ ASV to produce a bathymetric map; the AUV deployment with a predefined mission with areas of search based on the initial bathymetric map; and a final step performed by the MARES AUV to search for points of interest (missing workers, pipe structure, leak) in predefined search areas and collect visual inspection data.

For the initial survey and mapping, the ROAZ multibeam sonar is used. The vehicle builds in real time a coarse map and all the data is also logged allowing for postprocessing.

The vehicle navigation data (from the Advanced Navigation INS and Septentrio GPS fused data) is used to register the multibeam soundings. In real time, the vehicle has a $10 \mathrm{~cm}$ of position accuracy and less than $0.05^{\circ}$ in attitude accuracy. This data is then used in realtime to perform a fast statistical analysis in a $20 \mathrm{~cm}$ spatial grid (maximum, minimum and average measurements) in order to infer the bottom morphology.

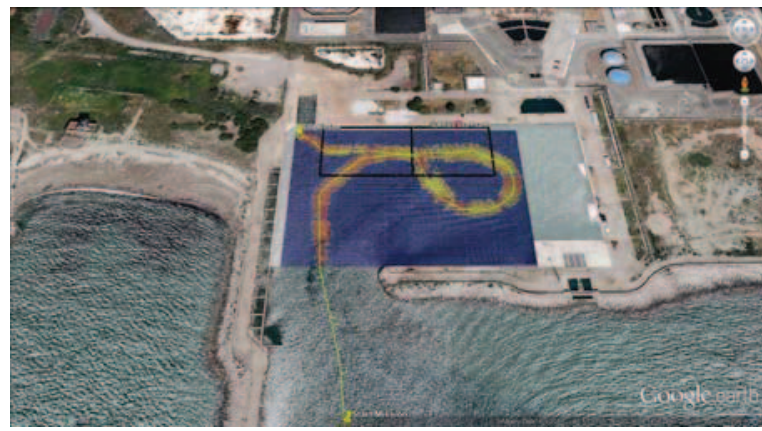

Fig. 5 - ROAZ trajectory and single pass realtime bathymetry survey along path superimposed in Google earth.

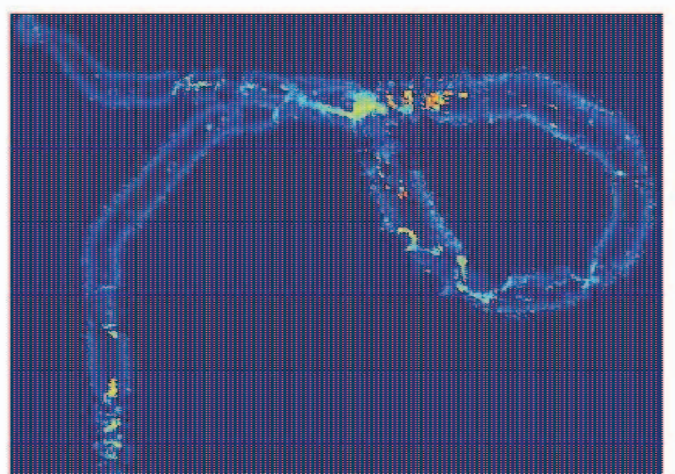

Fig 6. - Realtime multibeam sonar single track scan

This realtime map is the one used by the AUV path planner to define points of interest and search areas.

In post-processing, both the map build process and the navigation data accuracy can be increased providing a more accurate environment map.

Due to the limited operation space in relation with the ROAZ turning radius at the survey velocity $(3 \mathrm{~m} / \mathrm{s})$, and close proximity of the walls only a limited number of sweeps have been performed.

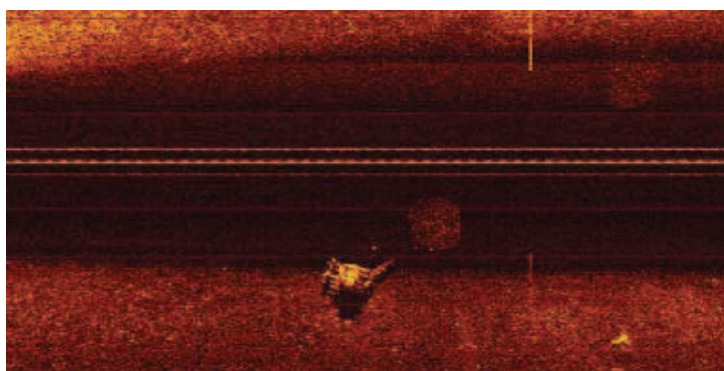

Fig 7 - Sidescan imaging of underwater structure

In addition, a sidescan sonar survey is also performed in the initial USV pass. This data can then provide imaging (Figure 7) of the sea bottom allowing for complementary environment assessment and identification of relevant features.

The use of an electrically powered vehicle with autonomous control also allows for reduced acoustic noise and greater trajectory control comparing with manned surveys.

\section{B. Autonomous AUV deploy}

One of the advantages of integration a surface and an underwater robot for search and rescue is the possibility of using the surface vehicle as a transport vessel to deploy (and recover) the AUV at a location of interest.

The autonomous deploy of the MARES AUV from ROAZ USV was tested at the euRathlon as a proof of concept.

A deploying mechanism was implemented on the starboard of ROAZ (Figure 8). 


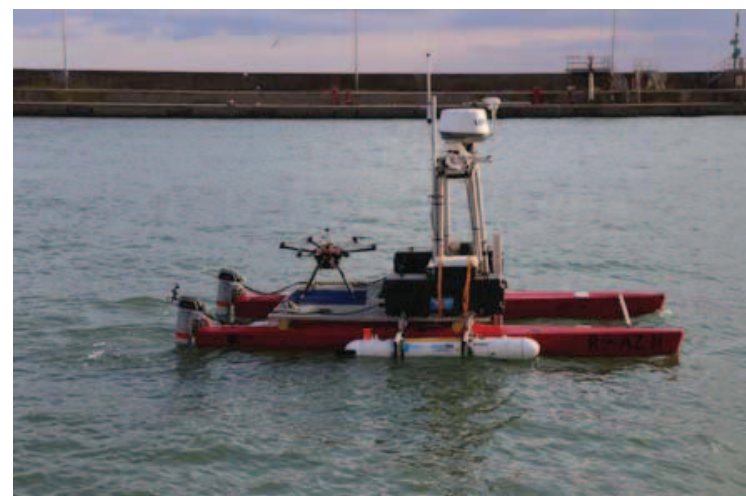

Fig. 8 - MARES AUV deploy setup from ROAZ USV

A predefined location as chosen for AUV deployment, and the surface robot autonomously navigated to that point deploying the AUV that then started its mission.

This autonomous deploy has the major advantage of allowing to preserve AUV energy (thus extending its operation autonomy) in the transport phase. In particular for areas of operation far from water access points, it provides crucial advantages, as the USV is capable of covering much larger distances, and eventually depending on the USV characteristics at faster speed. It also has the advantage of allowing for the AUV mission to be initialized close to the relevant area reducing the navigation errors incurred in long term underwater navigation.

The availability of an USV in the area also allows not only to provide a navigational acoustic aid but also to act as communication relay with underwater acoustic communications and above water radio communications to shore. The recovery of the AUV was not implemented due to mechanical restrictions and is an active area for research and development.

As seen in the Figure 8, there was also performed a liftoff test of an UAV from the USV (that further landed on land). This was done in context of the work being developed in order to integrate both air and underwater vehicles in cooperation with surface ones.

\section{Underwater search}

The underwater robot missions in the various challenges were focused on demonstrating autonomous underwater navigation capabilities, search and location of relevant points of interest, retrieving images from the search targets and underwater valve manipulation task.

The underwater search missions were planned taking into account the preliminary survey data obtained by the surface robot. Namely the expected depths and possible location for the possible targets (and bottom bathymetry) and the area to be searched. With this information the AUV path planner generated a lawnmown search trajectory at a convenient depth (in order to maximize the visual identification of features and avoiding possible obstacles).

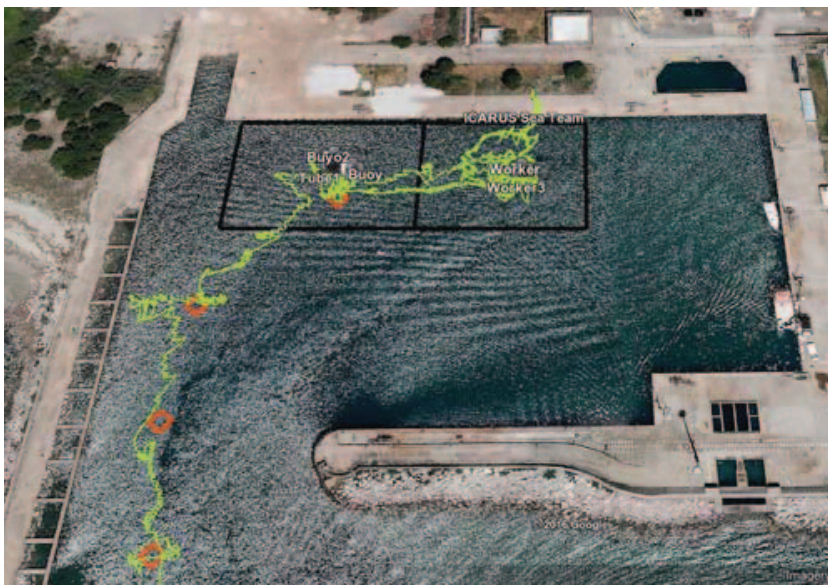

Fig. 9 - MARES AUV autonomous navigation trajectory (with required resurfacing waypoints and detected underwater targets location).

In Figure 9 one can observe the MARES trajectory in one of the trials (the Grand Challenge). In this case the AUV started in the first waypoint (orange mark) outside the harbor and was required to surface at each waypoint. The areas were already pre-surveyed by the USV and a mission was generate to search for the worker on the right arena. Following the worker search the planned mission followed to the left arena to search for the leaking pipe. This decision was made taking into account information received by the aerial robots that were inspecting the land build (and according to the rules were providing indications of in which underwater arena the leaking tube might be).

One can observe in the figure the detected locations of the missing worker and the leaking tube.

\section{On-board target detection}

The on-board visual target detection is color-based. All the relevant elements (piping, leaks and missing worker) had distinctive colors, either yellow or orange. In addition the pipes were numbered with large black numbers on the yellow surface. The valve was a horizontal bright pink cross.

Upon the image acquisition onboard, a color segmentation process was taken in order to detect possible targets. These were then classified according to their color and morphology.

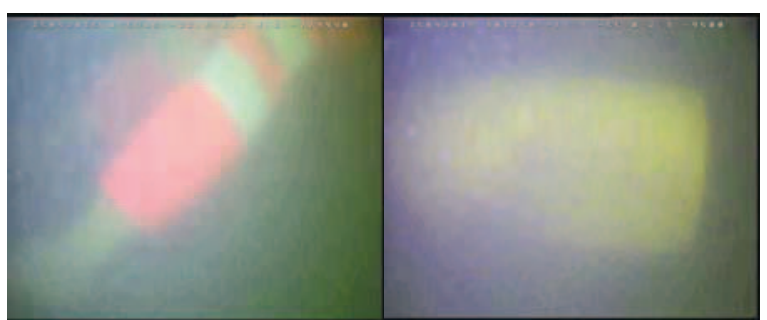

Fig. 10 - Underwater object of interest example images: victim (left), pipe entrance (right) 
In Figure 10, two images of detected targets are presented: the missing worker (on the left) and an underwater pipe. The AUV successfully achieved to locate more than $80 \%$ of the targets in all the missions even with the extremely low visibility conditions.

In particular the missing worker was a challenging task due to be in the middle of the water column and having smaller distinctive area (and easily confused with other scene elements such as buoys or the leaking marker). In this case and on the grand challenge mission, the cooperation with other robots of the team allowed for useful exchange of information narrowing the search.

\section{E. Underwater valve actuation}

The MARES AUV has a hovering capability due to its two vertical and horizontal thrusters. Due to this fact and although it does not possess a manipulator, a simple vertical pole was mounted in the front of the vehicle allowing for interaction with the underwater valve (in the challenge was implemented as a horizontal latch).

The valve actuation upon detection was to be performed as global motion of the vehicle moving forward and turning. During the tests the system was unable to turn the valve due to the reduced visibility coupled with the field of view of the camera. This resulted in the AUV losing the visual feedback from the valve very easily when moving in the approach to close the valve.

\section{CONCLUSIONS}

This work presented the multi-robot search and rescue missions performed in the context of the euRathlon 2015 robotic competition. The participation was integrated in the ICARUS multi-domain team that also included land and aerial robots.

An unmanned surface robot (ROAZ ASV) and an autonomous underwater vehicle (MARES AUV) were used to perform multiple tasks in the various marine related challenges of euRathlon. The surface robot was used to perform autonomously an initial large area survey, mapping and target identification and the AUV search mission was subsequently defined according to the results of the initial survey in order to provide precise location of targets (underwater pipe leaks of victims) and close inspection. Automatic target detection on the AUV was implemented and all the inspection and detection data was geo-referenced.

The USV successfully performed the autonomous navigation and mapping missions providing the initial data for the AUV search. The underwater visibility conditions were extremely bad (less than $1 \mathrm{~m}$, due to weather conditions) and even in these conditions, the team achieved excellent results both successfully detecting and identifying the leak, providing detailed mapping, infrastructure assessment and inspection visual data and successfully searching and locating the underwater victims. The global team participation also achieved the second place in the final multi-domain grand challenge.
An autonomous AUV deploy mission was also performed, allowing to validate autonomous transport and deployment in operational conditions.

Currently work is being developed towards the integrated and coordinated mission deployment and recovery of both AUV, and UAVs from the USV in order to provide additional situational awareness capabilities and provide support for navigation and communications to each robot.

\section{ACKNOWLEDGEMENTS}

The research leading to these results has received funding from the European Union Seventh Framework Programme (FP7/2007-2013) under grant agreement $\mathrm{n}^{\circ} 285417-$ ICARUS Project, and also from the ERDF - European Regional Development Fund through the Operational Programme for Competitiveness and Internationalisation COMPETE 2020 Programme within project «POCI-01-0145FEDER-006961», and by National Funds through the FCT Fundação para a Ciência e a Tecnologia (Portuguese Foundation for Science and Technology) as part of project UID/EEA/50014/2013.

\section{REFERENCES}

[1] De Cubber, G., Doroftei, D., Serrano, D., Chintamani, K., Sabino, R., \& Ourevitch, S., "The EU-ICARUS project: Developing assistive robotic tools for search and rescue operations.", 2013 IEEE International Symposium on Safety, Security, and Rescue Robotics (SSRR) , pp. 1-4, 2013

[2] Ferri, G., Ferreira, F., \& Djapic, V., "Boosting the talent of new generations of marine engineers through robotics competitions in realistic environments: The SAUC-E and euRathlon experience", Proc. IEEE\&MTS OCEANS 2015 - Genova ,pp. $1-6,2015$

[3] Murphy, R. R., "Findings from NSF-JST-NIST Workshop on Rescue Robotics", Proc. 2010 IEEE Safety Security and Rescue Robotics ,pp. 1-4, 2010

[4] Macwan, A., Nejat, G., \& Benhabib, B., "Optimal deployment of robotic teams for autonomous wilderness search and rescue". 2011 IEEE/RSJ International Conference on Intelligent Robots and Systems , pp. 4544-4549, 2011

[5] Ran, X., \& Ren, L., "Search Aid System Based on Machine Vision and Its Visual Attention Model for Rescue Target Detection", 2010 Second WRI Global Congress on Intelligent Systems, pp. 149-152, 2010

[6] Matos, A., Silva, E., Cruz, N., Alves, J. C., Almeida, D., Pinto, M., Machado, D., "Development of an Unmanned Capsule for large-scale maritime search and rescue", MTSS/IEEE OCEANS 2013, pp. 1-8. San Diego, California, USA, 2013

[7] Marconi, L., Melchiorri, C., Beetz, M., Pangercic, D., Siegwart, R., Leutenegger, S., Tomatis, N., "The SHERPA project: Smart collaboration between humans and ground-aerial robots for improving rescuing activities in alpine environments", 2012 IEEE International Symposium on Safety, Security, and Rescue Robotics (SSRR),pp. 1-4. IEEE, 2012 
[8] MIT, Ministry of Infrastructures and Transports - Italy. Marine Casualties Investigative Body - Costa Concordia Report, 2012

[9] Murphy, R. R., Steimle, E., Griffin, C., Cullins, C., Hall, M., \& Pratt, K., "Cooperative use of unmanned sea surface and micro aerial vehicles at Hurricane Wilma", Journal of Field Robotics, vol.25, n. 3, pp164-180, 2008

[10] Ohno, K., Kawatsuma, S., Okada, T., Takeuchi, E., Higashi, K., \& Tadokoro, S., "Robotic control vehicle for measuring radiation in Fukushima Daiichi Nuclear Power Plant", 2011 IEEE International Symposium on Safety, Security, and Rescue Robotics, pp. 38-43. IEEE, 2011

[11] Tadokoro, S., Kitano, H., Takahashi, T., Noda, I., Matsubara, H., Shinjoh, A. Shimada, S., "The RoboCup-Rescue project: a robotic approach to the disaster mitigation problem", Proceedings 2000 ICRA. Millennium Conference. IEEE International Conference on Robotics and Automation. Symposia Proceedings, vol. 4, pp. 4089-4094, 2000

[12] euRathlon consortium, euRathlon 2015 Challenge air + land + sea scenarios ver. 3.0, July, 2015

[13] Matos, A., Silva, E., Cruz, N., Alves, J. C., Almeida, D., Pinto, M., Machado, D., "Development of an Unmanned Capsule for large-scale maritime search and rescue", MTSS/IEEE OCEANS 2013, pp. 1-8, San Diego, California, USA,2013

[14] Martins, A., Dias, A., Almeida, J., Ferreira, H., Almeida, C., Amaral, G., Silva, E., "Field experiments for marine casualty detection with autonomous surface vehicles", MTS/IEEE OCEANS 2013, pp. 1-5, San Diego, CA, USA 